\section{ORIGINAL RESEARCH}
F. Al-Ali
T. Cree
S. Hall
S. Louis
K. Major

S. Smoker

S. Walker

\title{
Predictors of Unfavorable Outcome in Intracranial Angioplasty and Stenting in a Single-Center Comparison: Results from the Borgess Medical Center-Intracranial Revascularization Registry
}

\begin{abstract}
BACKGROUND AND PURPOSE: Intracranial angioplasty and stent placement are used to treat intracranial atherosclerotic disease. The 2 interventions have not been directly compared.

MATERIALS AND METHODS: This was a single-center, single-operator registry of consecutive, symptomatic subjects receiving treatment (angioplasty, BMS, or WS, chosen based on safety as judged by the operator). After November 2005, angioplasty alone was abandoned following the introduction of the WS. The primary end point was stroke rate per intervention at 30 days. The secondary end point was stroke rate per patient beyond 30 days. Success, dissection, restenosis, and occlusion rates were tracked.
\end{abstract}

\begin{abstract}
RESULTS: From April 2002 to January 2009, 140 subjects with 159 lesions (50\%-100\% stenosis) underwent 209 interventions: 89 angioplasty, 47 BMS, and 73 WS cases. Overall stroke rate at 30 days was $12.9 \%$. The angioplasty arm had the lowest stroke rate $(4.5 \%)$, whereas the WS arm had the highest $(24.7 \% ; P=.0002)$, leaving the BMS with $10.7 \%$. Stroke rate beyond 30 days was $9 \%$. The success rate was $58.4 \%$ for angioplasty, $81.3 \%$ for BMS, and $94.4 \%$ for WS, whereas the restenosis rates were $28.2 \%, 5.8 \%$, and $13.3 \%$, respectively. Dissection increased the risk of stroke in the first 30 days $(P=.0439)$ and restenosis $(P=.0051)$. Perforator vessels were more likely than nonperforators to have stroke within 30 days $(P=.008)$. Eccentric lesions were more likely to have stroke than concentric lesions $(P=.0726)$.
\end{abstract}

CONCLUSIONS: In this comparison, angioplasty had a significantly lower stroke rate than WS. Certain lesion locations, morphologic characteristics, and the presence of dissection after treatment were other predictors of unfavorable outcome.

\begin{abstract}
ABBREVIATIONS: $\mathrm{ACA}=$ anterior cerebral artery; $\mathrm{BA}=$ basilar artery; $\mathrm{BMC}$-IRR $=$ Borgess Medical Center-Intracranial Revascularization Registry; $B M S=$ balloon-mounted stent; $D C A=$ diagnostic cerebral angiogram; DES = drug-eluting stent; ICA = internal carotid artery; IRB = Institutional Review Board; $\mathrm{MCA}=$ middle cerebral artery; $\mathrm{mRS}=$ modified Rankin Scale; $\mathrm{TIA}=$ transient ischemic attack; $\mathrm{VA}=$ vertebral artery; VBJ = vertebrobasilar junction; WASID = Warfarin-Aspirin Symptomatic Intracranial Disease; WS = wingspan stent system
\end{abstract}

M ultiple intracranial angioplasty and stent placement series have been reported in the literature, with a wide range of complication rates. This is predominately due to their retrospective nature and the lack of standardized methods in reporting complications. More importantly, these series span long periods and are reported by different operators by using different techniques, making it virtually impossible to compare the safety and efficacy of different methods of endovascular revascularization.

\section{Materials and Methods}

\section{Study Design and Subject Eligibility}

The BMC-IRR was designed as a nonrandomized, single-center, single-operator, investigator-initiated data base. The protocol was

Received October 21, 2010; accepted after revision December 26.

From the Borgess Medical Center (T.C., S.W.), Borgess Research Institute, Kalamazoo, Michigan; Kalamazoo Center for Medical Studies (S.H.), Michigan State University, Kalamazoo, Michigan; and Neurointerventional Surgery and Diagnostic Services (F.A., S.L., K.M., S.S.), Borgess Medical Center, Kalamazoo, Michigan.

Please address correspondence to Firas Al-Ali, MD, Neurosurgery of Kalamazoo, 1541 Gull Rd, Suite 200, Kalamazoo, Ml 49048; e-mail: firasalali@aol.com

Indicates open access to non-subscribers at www.ajnr.org

DOI 10.3174/ajnr.A2530 approved by the local IRB, and all subjects gave consent for the procedure. IRB approval to waive informed consent for data collection and analysis was obtained. Data were entered prospectively and retrospectively in a secure data base (MD Analyze; Medtech Global, South Melbourne, Vic, Australia; www.medtechglobal.com) and analyzed retrospectively. Subjects were included in the registry if they were $\geq 40$ years of age and presented with TIA or stroke ( $>1$ day old) that was attributable to angiographically verified $\geq 50 \%$ arterial stenosis. Exclusion criteria were contraindication to aspirin or to clopidogrel (Plavix).

$\mathrm{mRS}^{1}$ was documented directly or inferred from the subject's chart before treatment and before discharge. All subjects were on aspirin and clopidogrel before intervention, per protocol, and stayed on dual-antiplatelet therapy for at least 3 months (6 months if treated with a DES). In 2007, dual-antiplatelet therapy was prolonged to 1 year in subjects with DES after 1 patient experienced delayed stent thrombosis. The protocol called for a follow-up DCA at 3 months (6 months for DES) and an office visit at 1 year, and $\mathrm{mRS}$ was repeated each time.

\section{Endovascular Revascularization Technique}

All subjects were placed on $81 \mathrm{mg}$ of aspirin and $75 \mathrm{mg}$ of clopidogrel for at least 5 days before intervention. In cases where intervention was 
planned within 24 hours for subjects who were not previously on clopidogrel, a loading dose of $300 \mathrm{mg}$ was given orally at least 12 hours before the procedure. From April 2002 to June 2007, most posterior circulation interventions were performed under general anesthesia, whereas most anterior circulation lesions were treated under conscious sedation. Starting in June 2007, almost all interventions were performed under general anesthesia. Overall, 132 of 209 interventions (63\%) were performed under general anesthesia.

Special care was taken to place the guiding catheter as close to the lesion as possible (a task greatly facilitated by the introduction of the Neuron Guiding Catheter; Penumbra, San Leandro, California). After giving the subjects $5000 \mathrm{U}$ intravenous heparin bolus, activated clotting time was maintained at 200-250 seconds throughout the procedure by subsequent administration of intravenous heparin bolus. Measurement of activated clotting time was repeated every $20-$ 30 minutes. The lesion was then crossed by using a soft-tip 0.014 -inch outer diameter 180-mm microwire (Transend; Boston Scientific, Natick, Massachusetts) if the subject was to undergo angioplasty or BMS. If WS was contemplated, a 300-mm exchange-length microwire was used (Boston Scientific). The device was advanced to the lesion over the guidewire, and the balloon was gradually and slowly inflated over a period of 2-3 minutes before reaching nominal pressure for the angioplasty cases, and 20-30 seconds for the BMS cases. Care was taken to undersize the device by at least $0.5 \mathrm{~mm}$ of the normal vessel diameter. The balloon catheter was then pulled back a few centimeters and, with the microwire still in place, a follow-up angiogram was obtained through the guiding catheter to confirm adequate results, rule out significant complications (such as ruptured vessels), and assess the presence of dissection. With the WS, the balloon catheter was pulled out, and the stent system was deployed over the pre-existing exchange-length microwire covering the angioplasty site. The stent delivery catheter was then removed and a repeat angiogram was obtained to confirm adequate expansion of the stent. If the stent was not sufficiently expanded ( $\geq 50 \%$ residual stenosis), a new balloon catheter $(0.5 \mathrm{~mm}$ larger than the first $)$ was advanced over the pre-existing microwire, and a repeat angioplasty was performed. A repeat angiogram was obtained after pulling out the balloon catheter, to confirm adequate expansion of the stent and to rule out delayed clot formation. If the last angiogram revealed no abnormalities, it was repeated 5 minutes later, and if no complications were identified the microwire and balloon catheter were removed. Heparin was not reversed. Subjects were kept in the intensive care unit for 24 hours, after which they were discharged to home or to the rehabilitation unit, depending on their clinical status.

\section{Vessel Treated and Device Used}

The ICA, the MCA, and the VA were defined according to customary medical definitions. However, the VBJ was considered part of the BA. Vessels with no perforators originating from them (ICA and VA) were classified as nonperforator vessels, whereas vessels with perforators originating from them (MCA and BA) were classified as perforator vessels.

The choice to use 1 of the 3 treatment options (angioplasty, BMS, or WS) was based on the safety of the device as determined by the operator for each particular patient. Before the introduction of WS (November 2005), angioplasty was favored over BMS for vessels with marked tortuosity (MCA and ICA) or perforators due to the stiffness of the BMS and its inherent difficulty to navigate through multiple curves. BMS was used for vessels with few curves between the tip of the guiding catheter and the lesion (2-3 curves), especially for lesions in straight vessels, such as the basilar artery or petrous ICA. For vessels with marked angulation, such as the MCA, angioplasty was favored to minimize the potential straightening and ensuing rupture of perforators if we deployed a stiff device such as a BMS. When BMS was chosen, vessel diameter dictated the type of stent. For vessels with a caliber $<3.5 \mathrm{~mm}$ (VA and BA), a DES was used. With the introduction of the WS, angioplasty was almost entirely replaced by WS, except in vessels $\leq 2 \mathrm{~mm}$ in diameter.

\section{Lesion Morphology}

Lesion morphology was characterized at first presentation only (primary lesion) as smooth versus irregular, concentric versus eccentric, and by length as short $(<5 \mathrm{~mm})$, moderate $(5-10 \mathrm{~mm})$, or long $(>10 \mathrm{~mm})$.

\section{Eligibility for Retreatment}

The indication for retreatment was either a finding of significant restenosis $(\geq 70 \%)$ on follow-up DCA or a recurrence of symptoms as reported by the patient and confirmed by the physician during an office consultation.

\section{Outcome Measurements}

Stroke rate per intervention (all cases combined: primary intervention as well as cases of restenosis) within the first 30 days was the primary end point and was classified as major if a change in the $\mathrm{mRS}$ from baseline was documented as $\geq 2$. If the changes were $\leq 1$, the stroke was classified as minor. Stroke rate per patient beyond the first 30 days was the secondary end point. A stroke during this period was attributed to the intervention immediately preceding its occurrence.

Success rate was based on intention to treat, and success was defined as $\leq 50 \%$ residual stenosis and no unplanned intervention. Dissection rate was defined as a clear flap, or diffuse haziness and decrease of opacity found in the treated segment on angiography immediately after the intervention. Restenosis rate was defined as $\geq 70 \%$ stenosis on follow-up DCA within the stipulated timeframe of angiographic follow-up. The choice of $\geq 70 \%$ stenosis matches the criterion used in deciding to retreat primary lesions.

Overall vascular occlusion rate was based on the number of primary lesions that were treated and had angiographic follow-up. Two lesions treated with a DES came back with massive stroke on CT scan, involving the whole territory of the stented vessel. These cases were counted as occlusion despite the absence of an angiographic study. Device occlusion rate was considered per intervention, and occlusion was attributed to the last device used.

We also performed a second set of analysis involving all primary lesions, with morphologic characteristic available, after the first intervention. Stroke rate at 30 days and success rate after intervention (by using the same definition as given above) were calculated. Restenosis rate and occlusion rate at their follow-up catheter angiogram also were calculated before any other intervention if it occurred. All of these outcome measures were correlated with the primary lesion morphology (smooth versus irregular, concentric versus eccentric, and short versus long lesions).

\section{Statistical Analyses}

Age, sex, and lesion morphology data were summarized for each patient. Lesions and interventions were individually characterized. Proportions among pair-wise comparisons were analyzed by using a 2-proportion $Z$-test. $\chi^{2}$ and Mantel-Haenszel $\chi^{2}$ tests were performed 


\begin{tabular}{|c|c|c|c|}
\hline & \multicolumn{3}{|c|}{ Balloon-Mounted } \\
\hline & Angioplasty & Stent & Wingspan Stent \\
\hline Mean age (yr) & 64.6 & 69.1 & 66.1 \\
\hline Male/female distribution by 159 lesions & $26 / 29$ & $31 / 8$ & $37 / 28$ \\
\hline \multicolumn{4}{|l|}{ Presentation } \\
\hline Mean days from first symptom to treatment & 69.3 & 48.5 & 61.0 \\
\hline Mean presenting $\mathrm{mRS}$ & 1.5 & 1.4 & 1.6 \\
\hline Mean stenosis before treatment of 159 primary lesions (\%) & 83 & 77 & 78 \\
\hline Transient ischemic attack $(n=61)$ & 19 & 19 & 23 \\
\hline Minor stroke (change of $0-1$ in $\mathrm{mRS}, n=45$ ) & 20 & 9 & 16 \\
\hline Major stroke (change of $\geq 2$ in $\mathrm{mRS}, n=52$ ) & 15 & 11 & 26 \\
\hline \multicolumn{4}{|l|}{ Vessel and lesion characteristics for all 159 lesions } \\
\hline Lesions per device & 55 & 39 & 65 \\
\hline$<70 \%$ stenosis & 2 & 4 & 7 \\
\hline$\geq 70 \%$ stenosis & 53 & 35 & 58 \\
\hline Perforators & 32 & 13 & 47 \\
\hline Nonperforators & 23 & 26 & 18 \\
\hline Smooth lesion & 31 & 27 & 41 \\
\hline Irregular lesion & 17 & 12 & 20 \\
\hline Concentric lesion & 20 & 16 & 41 \\
\hline Eccentric lesion & 28 & 23 & 20 \\
\hline Short lesion (<5 mm) & 19 & 18 & 22 \\
\hline Moderate lesion $(5-10 \mathrm{~mm})$ & 6 & 11 & 10 \\
\hline Long lesion (>10 mm) & 23 & 10 & 29 \\
\hline \multicolumn{4}{|l|}{ Vessel and lesion characteristics for all 209 interventions } \\
\hline Interventions per device & 89 & 47 & 73 \\
\hline$<70 \%$ stenosis & 9 & 5 & 8 \\
\hline$\geq 70 \%$ stenosis & 79 & 43 & 65 \\
\hline Perforators & 51 & 14 & 54 \\
\hline Nonperforators & 38 & 33 & 19 \\
\hline Smooth lesion & 57 & 33 & 46 \\
\hline Irregular lesion & 20 & 15 & 22 \\
\hline Concentric lesion & 40 & 21 & 46 \\
\hline Eccentric lesion & 37 & 27 & 22 \\
\hline Short lesion $(<5 \mathrm{~mm})$ & 26 & 20 & 22 \\
\hline Moderate lesion (5-10 mm) & 9 & 11 & 10 \\
\hline Long lesion $(>10 \mathrm{~mm})$ & 42 & 17 & 36 \\
\hline Activating clotting time at deployment (s) & 212 & 212 & 217 \\
\hline Duration of procedure (min) & 163 & 149 & 154 \\
\hline
\end{tabular}

to analyze the 2-by-2 tables. Descriptive statistics were calculated as well. The $\alpha$ value used to determine statistical significance was 0.10 . There were no multiple comparisons adjustments performed.

\section{Results}

Between April 2002 and January 2009, 140 subjects with 159 lesions (50\%-100\% stenosis, Table 1) underwent 209 interventions. Of the 209, 21 were for 50\%-69\% stenosis. One intervention was performed for $<50 \%$ stenosis and was undertaken because a large ulcer was felt to be the cause of symptoms and was grouped with the 50\%-69\% stenosis group; resulting in a total of 22 interventions with $50 \%-69 \%$ stenosis. The remaining 187 interventions $(89.5 \%)$ were performed for $\geq 70 \%$ stenosis.

In total, 139 patients with 158 lesions were symptomatic, and 1 patient with 1 lesion was found incidentally and treated due to the degree of stenosis. Of the lesions, 61 presented with TIA and 97 with stroke; 45 strokes were minor and 52 were major. Of the 158 lesions, there were 10 that we could not confirm as the source of the patient's presenting symptoms (ie, the patient had other contributing factors, such as aortic plaque, a cardiac source of emboli, or carotid bifurcation dis- ease). Of the 209 interventions, 89 were with angioplasty, 47 with BMS, and 73 with WS. Demographics, clinical presentation, lesion location, and morphologic characteristics were similar in the 3 groups, with the exception of low presentation of perforator vessels in the BMS arm (Table 1).

Of the 140 subjects, 133 (95\%) had clinical follow-up of $>1$ month. Three patients died in the first 30 days postprocedure, 1 patient due to the presenting stroke, 1 patient due to vessel rupture during the intervention, and 1 patient due to organ failure. Four patients were lost to follow-up. Mean clinical follow-up time was 26 months (1-88 months). One hundred and three patients had clinical follow-up $>1$ year, with a mean of 31 months. Follow-up vascular imaging was performed for 183 of 209 interventions (87.5\%). Follow-up DCA was done for 171 interventions, MR angiogram for 9, and CT angiogram for 3. One hundred sixty five of the 209 interventions had a DCA within the stipulated timeframe of 3 months (6 months for DES).

Of the 159 primary lesions, 148 had morphologic characteristics available (7 had total $100 \%$ occlusion, making it impossible to evaluate the plaque; in the other 4 lesions, we were 
Table 2: Outcomes of intracranial revascularization by type of intervention

\begin{tabular}{|c|c|c|c|c|c|c|c|}
\hline & $\begin{array}{c}\text { All } \\
\text { Interventions } \\
\text { No. }(\%)\end{array}$ & $\begin{array}{c}\text { Angioplasty } \\
\text { No. }(\%)\end{array}$ & $\begin{array}{l}\text { Angioplasty vs } \\
\text { Balloon-Mounted } \\
\text { Stent } P \text { Value }\end{array}$ & $\begin{array}{c}\text { Balloon-Mounted } \\
\text { Stent } \\
\text { No. }(\%)\end{array}$ & $\begin{array}{c}\text { Balloon-Mounted } \\
\text { Stent vs } \\
\text { Wingspan } \\
P \text { Value }\end{array}$ & $\begin{array}{l}\text { Wingspan } \\
\text { Stent } \\
\text { No. }(\%)\end{array}$ & $\begin{array}{l}\text { Wingspan } \\
\text { Stent vs } \\
\text { Angioplasty } \\
P \text { Value }\end{array}$ \\
\hline Stroke at 30 days & $27 / 209(12.9)$ & $4 / 89(4.5)$ & .1721 & $5 / 47(10.7)$ & .0579 & $18 / 73(24.7)$ & .0002 \\
\hline Major & $10 / 209(4.8)$ & $2 / 89(2.25)$ & & $2 / 47(4.3)$ & & 6/73 (8.3) & \\
\hline Minor & $17 / 209(8.1)$ & $2 / 89(2.25)$ & & $3 / 47(6.4)$ & & $12 / 73(16.4)$ & \\
\hline Stroke beyond 30 days & $12 / 133(9.0)$ & $3 / 45(6.7)$ & .3802 & $4 / 32(12.5)$ & .5969 & $5 / 56(8.9)$ & .6772 \\
\hline Success by intervention & $159 / 209(76.1)$ & $52 / 89(58.4)$ & .0039 & $39 / 48(81.3)$ & .0233 & $68 / 72(94.4)$ & $<.0001$ \\
\hline Restenosis & $30 / 165(18.2)$ & $20 / 71(28.2)$ & .0082 & $2 / 34(5.8)$ & .2628 & $8 / 60(13.3)$ & .0358 \\
\hline Device occlusion by intervention & $10 / 189(5.3)$ & $4 / 83(4.8)$ & .3657 & $4 / 45(8.9)$ & .2188 & 2/61 (3.3) & .6487 \\
\hline Dissection by intervention & $46 / 209(22.0)$ & $17 / 89(19.1)$ & .7667 & $8 / 47(17.0)$ & .1440 & $21 / 73(28.8)$ & .1498 \\
\hline
\end{tabular}

Table 3: Incidence of stroke at $\mathbf{3 0}$ days by intervention and lesion location

\begin{tabular}{|c|c|c|c|c|}
\hline & $\begin{array}{c}\text { Total No. } \\
(\%)\end{array}$ & $\begin{array}{c}\text { Angioplasty } \\
\text { No. }(\%)\end{array}$ & $\begin{array}{l}\text { Iloon-Mount } \\
\text { Stent } \\
\text { No. }(\%) \\
\end{array}$ & $\begin{array}{c}\text { Wingspan Stent } \\
\text { No. }(\%)\end{array}$ \\
\hline \multicolumn{5}{|l|}{ Vessel } \\
\hline ACA/MCA & $13 / 80(16.3)^{\mathrm{a}}$ & $3 / 44(6.8)$ & $0 / 1(0)$ & $10 / 35(28.6)$ \\
\hline ICA & $2 / 41(4.9)^{a, b}$ & $0 / 16(0)$ & $1 / 14(7.1)$ & $1 / 11(9.1)$ \\
\hline VA & $4 / 48(8.3)^{c}$ & $1 / 21(4.8)$ & $1 / 19(5.3)$ & $2 / 8(25.0)$ \\
\hline $\mathrm{BA}$ & $8 / 40(20.0)^{b}$ & $0 / 7(0)$ & $3 / 14(21.4)$ & $5 / 19(26.3)$ \\
\hline \multicolumn{5}{|l|}{ Circulation } \\
\hline Anterior & $15 / 121(12.4)$ & $3 / 60(5.0)$ & $1 / 15(6.7)$ & $11 / 46(23.9)$ \\
\hline Posterior & $12 / 88(13.6)$ & $1 / 28(3.6)$ & $4 / 33(12.1)$ & $7 / 27(25.9)$ \\
\hline \multicolumn{5}{|l|}{ Perforator status } \\
\hline Nonperforator (VA, ICA) & $6 / 91(6.6)^{d}$ & $1 / 39(2.6)$ & $2 / 33(6.1)$ & $3 / 19(15.8)$ \\
\hline Perforator (BA, MCA) & $21 / 118(17.8)^{d}$ & $3 / 49(6.1)$ & $3 / 15(20.0)$ & $15 / 54(27.8)$ \\
\hline Total & $27 / 209(12.9)$ & $4 / 89(4.5)$ & $5 / 47(10.7)$ & $18 / 73(24.7)$ \\
\hline
\end{tabular}

aCA versus ICA: $P=.0736$.

ICA versus $B A: P=.0398$.

c VA versus all vessels: $P=\mathrm{NS}$.

${ }^{\mathrm{d}}$ Nonperforator versus perforator: $P=.008$.

not sure whether they had smooth plaque or a partially recanalized clot).

\section{Primary and Secondary End Points}

Stroke within 30 days, the primary end point, occurred in $12.9 \%$ of interventions (Table 2). Of these, $4.8 \%$ were major strokes and $8.1 \%$ were minor. Patients treated with angioplasty had the lowest stroke rate at 4.5\% (2.25\% major, 2.25\% minor), whereas WS patients had the highest stroke rate at $24.7 \%$ (8.3\% major, $16.4 \%$ minor), and BMS patients had an intermediate rate of $10.7 \%$ ( $4.3 \%$ major, $6.4 \%$ minor). There was no statistical difference for the odds of stroke for subjects receiving angioplasty versus BMS, but the odds were significantly higher for WS versus angioplasty $(P=.0002)$ and WS versus BMS $(P=.0579)$.

Analyses of the interaction between lesion location and chance of stroke within the first 30 days are shown in Table 3. There was 1 intervention on the ACA; for statistical purposes, it was grouped with the MCA. The stroke rate in vessels with perforators was almost 3 -fold that of vessels without perforators for all treatments $(P=.008)$. Of importance, there was no statistical difference between anterior versus posterior circulation or when comparing the VA to any other vessel. Comparisons of stroke rate between the MCA and ICA $(P=.0736)$ as well as the BA and ICA $(P=.0398)$ were significantly different.

Stroke at last follow-up of $>1-88$ months, the secondary end point, occurred in $9 \%$ of patients (Table 2). It was lowest
Table 4: Influence of dissection and specific lesion morphology on incidence of stroke and restenosis

\begin{tabular}{|c|c|c|}
\hline & $\begin{array}{c}\text { Stroke within } \\
30 \text { Days for } \\
209 \text { Interventions } \\
\text { No. }(\%)\end{array}$ & $\begin{array}{c}\text { Restenosis } \\
\text { on } 165 \text { Follow-Up } \\
\text { Angiograms for } \\
209 \text { Interventions } \\
\text { No. }(\%)\end{array}$ \\
\hline Dissection & $10 / 46(22)$ & $13 / 39(33)$ \\
\hline No dissection & $17 / 163(10)$ & $17 / 126(14)$ \\
\hline Significant $P$ value & .0439 & .0051 \\
\hline Smooth/concentric/long lesions & $4 / 43(9)$ & $17 / 30(56.7)$ \\
\hline All other morphology & $23 / 166(14)$ & $13 / 135(10)$ \\
\hline Significant $P$ value & NS & $<.0001$ \\
\hline
\end{tabular}

in the angioplasty arm (6.7\%), highest in the BMS arm (12.5\%), and intermediate in the WS arm (8.9\%); no difference was statistically significant.

\section{Success, Restenosis, and Occlusion Rates}

Overall success rate was $76 \%$ (Table 2). The angioplasty arm had the lowest success rate $(58.4 \%)$, whereas the WS arm had the highest (94.4\%), and the BMS arm fell in between (81.3\%). The overall restenosis rate was $18.2 \%$. The angioplasty arm had the highest rate $(28.2 \%)$, followed by the WS arm (13.3\%) and the BMS arm (5.8\%). There was a statistically significant difference in restenosis between the angioplasty arm and the BMS arm $(P=.0082)$ and between the angioplasty arm and the WS $\operatorname{arm}(P=.0358)$. Of importance, lesion location was not a predictor of restenosis. 
Specific lesion morphology was a predictor of restenosis (Table 4). Of the 30 lesions with restenosis, 25 were long $(83.3 \%), 26$ were smooth $(86.6 \%)$, and 20 were concentric $(66.6 \%)$, and 17 were smooth, concentric, and long $(56.7 \%)$. Of the 43 lesions that were smooth, concentric, and long before intervention, 35 had a follow-up DCA at the stipulated time and 24 of those 35 had restenosis (68.5\%). Restenosis was significantly more likely in smooth, concentric, and long lesions $(P<.0001)$ than in other types of lesions.

Vascular occlusion rate was $6.5 \%(10 / 152)$. Device occlusion rate was $5.3 \%(10 / 189 ;$ Table 2$)$. The WS arm had the lowest rate $(3.3 \%)$ and the BMS arm had the highest $(8.9 \%)$, with the angioplasty arm midway (4.8\%). No comparisons were statistically significant. Occlusions in the WS and angioplasty arms were clinically silent, whereas the 4 occlusions in the BMS arm were all symptomatic. Two resulted in massive stroke and death, whereas the other 2 patients had minor strokes.

\section{Dissection}

Dissection was found in $22 \%$ of interventions (Table 2). The BMS arm had the lowest dissection rate $(17.0 \%)$, whereas the WS arm had the highest $(28.8 \%)$. The angioplasty arm fell in between $(19.1 \%)$, and there was no statistical significance in dissection rate between treatment arms. Dissection was a significant predictor of stroke at 30 days $(P=.0439)$ and $\geq 70 \%$ restenosis at angiographic follow-up $(P=.0051$; Table 4$)$.

\section{Primary Lesion Morphology and Outcome Measures}

Of the 148 primary lesions with morphologic changes available, stroke within 30 days, the primary end point, occurred in $16 \%$ of interventions. None of the lesion morphology characteristics were statistically significant in predicting outcome. The overall success rate was $80 \%$. Of the morphologic characteristics, only smooth lesions had a significantly higher odds of success compared with irregular lesions $(P=.078)$. Restenosis rate, however, was associated with long and smooth lesions ( $P=.008$ and $P=.024$, respectively), which echoes the finding of the whole group as mentioned above. No statistically significant association was found between the primary lesion morphology and the occlusion rate (Table 4).

\section{Discussion}

\section{Angioplasty versus Stenting: Primary and Secondary End Points}

To our knowledge, this study represents the first time that angioplasty and stent placement have been directly compared. Our registry is well suited to make this direct comparison, especially between the angioplasty and the WS arms, because those patients had similar demographics, lesion distributions, and the same treatment protocol. Furthermore, our complication rate with each device is close to that reported in the literature, with the apparent exception of the WS group.

Several angioplasty series report a wide range of complication rates from $4 \%$ to $40 \% .^{2-5}$ Our angioplasty stroke rate at 30 days $(4.5 \%)$ is closer to the lower end of this range. For BMS, again our stroke rate at 30 days $(10.7 \%)$ is well within the reported range of $6 \%-28.5 \% .^{6-9}$ Our results actually compare favorably with the latest series for BMSs, which reports a complication rate of $28.5 \% .^{7}$

The reported WS series deserves closer examination. The Wingspan study ${ }^{10}$ had a $4.5 \%$ complication rate at 30 days. Upon closer analysis, there were 18 minor adverse events in 12 subjects; at least 2 of these could be classified as minor stroke (frontal medial branch occlusion and nystagmus), bringing the 30-day stroke/death rate to 9.1\% (4/44). An additional stroke occurred during the follow-up period, for a total stroke/death rate within 6 months of $11.3 \%$ (5/44). This is almost identical to the $11 \%$ stroke rate reported in the first year of the WASID study. ${ }^{11}$ A later article reported a $6.1 \%$ major complication rate at 30 days. ${ }^{12}$ There was no mention of minor complications, including minor stroke, making it difficult to determine the real stroke rate, but it is very close to our reported major stroke rate in the WS arm of $8.3 \%$.

In addition, the National Institutes of Health registry report included 6-month clinical follow-up, ${ }^{13}$ with a stroke rate/ death of $14 \%$ (6.2\% at 24 hours, $9.6 \%$ at 30 days, and $14 \%$ at 6 months). Of importance, when the authors divided the centers by high- $(>8$ cases per site) and low-enrollment sites $(<8$ cases per site), they found that the stroke rate varied significantly, with $26.9 \%$ stroke/death rate in low-enrollment sites versus $9.5 \%$ at high-enrollment sites. The authors explained this discrepancy by suggesting that the operators in the lowenrollment sites had less experience with the device and expected their complication rate to drop once their experience increased. Although this explanation is logical, we believe it is somewhat problematic. First, the operators at the lowenrollment sites, though new to the device, were by no means novice operators, and most of them had performed more complicated procedures in the past. Second, it should not take 8 cases for operators to significantly decrease their complication rate from $26.9 \%$ to $9.5 \%$. Comparing this series with BMC-IRR is intersecting, because the authors report the stroke rate in similar way to us, and our registry has a very close stroke rate to their supposedly low-enrollment site. Our complications (strokes) did not cluster at the beginning of WS use; rather, they stretched along the whole period, making the lack of experience explanation less likely.

Our direct comparison has limitations because the devices were not compared with each other in a randomized manner. Because safety was our major concern, bias was introduced from the beginning by selecting the safest device in the operator's opinion. This particular bias, however, was equally introduced to all 3 devices; and, if anything, it artificially decreased the odds of stroke in the BMS arm because we rarely used BMS in vessels with perforators, which we have shown carry a higher risk of stroke after intervention. Therefore, our statistical difference in stroke rate at 30 days between BMS and WS $(P=.0579)$ is ambiguous and may not be reproducible in a head-to-head comparison. Our comparison of angioplasty to WS, however, is stronger because both arms share a similar lesion distribution, degree of stenosis, and the same technique.

Interestingly, angioplasty held its advantage over either stent when evaluating stroke beyond 30 days. The difference in stroke beyond 30 days between angioplasty (6.7\%) and WS $(8.9 \%)$ is minor. However, the $12.5 \%$ stroke rate for BMS is almost double that of angioplasty, albeit not statistically significant, and was due to the higher incidence of delayed 
thrombosis, which could be due to the drug-eluting nature of the stent we used (paclitaxel). Further study is warranted.

\section{Other Predictors of Outcome}

Lesion Location. Contrary to other reports, ${ }^{14}$ we found no statistically significant difference in stroke rate at 30 days between anterior and posterior circulation. Vessels with perforators, however, had a statistically higher stroke rate than vessels without perforators. The difference between vessels with and without perforators is new, but agrees with previous reports of higher complication rates in the BA compared with the VA. ${ }^{15}$ This is an important observation, because the WASID study demonstrated an equal risk of stroke after treatment, independent of the vessel location. ${ }^{11}$ Because angioplasty had a low and acceptable stroke rate in perforator vessels, a practical approach could be to use angioplasty as a first line to treat these vessel lesions and to reserve stents as a salvage device. Today in our practice, we consider angioplasty as the first line of treatment for patients with perforators. WS is used only when we are faced with large dissection. Those patients also will have an extended length of stay, at least 48 hours, for close monitoring.

Dissection. Dissection was a strong predictor of 2 unfavorable outcomes that have not been recognized before: a higher stroke rate at 30 days and a higher restenosis rate on follow-up DCA. We believe if dissection is present after intervention, then extra measures should be taken, such as extending the length of stay. This observation still requires confirmation by another investigator, a larger series of study, or both.

Lesion Morphology. Of all morphologic characteristics, only eccentric versus concentric lesions was a predictor of stroke at 30 days $(P=.0726)$. Although short lesions had almost half the stroke rate compared with moderate or long lesions, it was not statistically significant. This is in agreement with the Mori classification. ${ }^{16}$ The short concentric lesion in BMC-IRR corresponds very closely to the Mori type "A" lesion with the lowest complication rate in both series. We did not compare our lesions to Mori type B and C, because we found these, with the exception of occlusion classification, very subjective and difficult to replicate. But we agree that lesions with $100 \%$ stenosis (occlusion) carry a significantly higher risk of intervention. Interestingly, specific lesion morphology was a predictor of restenosis; smooth and long lesions were highly associated with restenosis. To our knowledge, this finding has not been reported previously.

\section{Conclusions}

In this comparison, angioplasty was the safest technique of endovascular intracranial revascularization and was signifi- cantly safer than WS. Lesion location (perforator vessels), morphology (eccentric lesions), and the presence of dissection after treatment were also predictors of unfavorable clinical outcome. Lesion location was not a predictor of restenosis, but the presence of dissection after intervention and specific lesion morphology (smooth and long) were predictors.

\section{Acknowledgments}

We thank Sarah Staples, MA, ELS and Anne Jefferson, BA (candidate) for assisting with manuscript preparation.

\section{References}

1. Bonita R, Beaglehole R. Modification of Rankin Scale: recovery of motor function after stroke. Stroke 1988;19:1497-500

2. Sundt TM, Smith HC, Campbell JK, et al. Transluminal angioplasty for basilar artery stenosis. Mayo Clin Proc 1980;55:673-80

3. Derdeyn CP, Chimowitz MI. Angioplasty and stenting for atherosclerotic intracranial stenosis: rationale for a randomized clinical trial. Neuroimaging Clin N Am 2007;17:355-63

4. Connors JJ, Wojak JC. Percutaneous transluminal angioplasty for intracranial atherosclerotic lesions: evolution of technique and short-term results. J Neurosurg 1999;91:415-23

5. Marks MP, Wojak JC, Al-Ali F, et al. Angioplasty for symptomatic intracranial stenosis: clinical outcome. Stroke 2006;37:1016-20

6. du Mesnil de Rochemont R, Turowski B, Buchkremer M, et al. Recurrent symptomatic high-grade intracranial stenoses: safety and efficacy of undersized stents-initial experience. Radiology 2004;231:45-49

7. Stenting of symptomatic atherosclerotic lesions in the vertebral or intracranial arteries (SSYLVIA): study results. Stroke 2004;35:1388-92

8. Kurre W, Berkefeld J, Sitzer M, et al. Treatment of symptomatic high-grade intracranial stenoses with the balloon-expandable Pharos stent: initial experience. Neuroradiology 2008;50:701-08

9. Mazighi M, Yadav JS, Abou-Chebl A. Durability of endovascular therapy for symptomatic intracranial atherosclerosis. Stroke 2008;39:1766-69

10. Bose A, Hartmann M, Henkes $\mathrm{H}$, et al. A novel, self expanding, nitinol stent in medically refractory intracranial atherosclerotic stenoses. The Wingspan study. Stroke 2007;38:1531-37

11. Chimowitz MI, Lynn MJ, Howlett-Smith H, et al. for the Warfarin-Aspirin Symptomatic Intracranial Disease Trial Investigators. Comparison of warfarin and aspirin for symptomatic intracranial arterial stenosis. $N \mathrm{Engl} \mathrm{J} \mathrm{Med}$ 2005;352:1305-16

12. Fiorella D, Levy EI, Turk AS, et al. US multicenter experience with the Wingspan stent system for the treatment of intracranial atheromatous disease: periprocedural results. Stroke 2007;38:881-87

13. Zaidat OO, Klucznik R, Alexander MJ, et al for the NIH Multi-center Wingspan Intracranial Stent Registry Study Group. The NIH registry on use of the Wingspan stent for symptomatic $70-99 \%$ intracranial arterial stenosis. Neurology 2008;70:1518-24

14. Gröschel K, Schnaudigel S, Pilgram SM, et al. A systematic review on outcome after stenting for intracranial atherosclerosis. Stroke 2009;40:e340-47

15. Jiang WJ, Xu XT, Du B, et al. Long-term outcome of elective stenting for symptomatic intracranial vertebrobasilar stenosis. Neurology 2007;68: $856-58$

16. Mori T, Fukuoka M, Kazita K, et al. Follow-up study after intracranial percutaneous transluminal cerebral balloon angioplasty. AJNR Am J Neuroradiol 1998;19:1525-33 